\title{
MicroRNA-218 inhibits tumor angiogenesis of human renal cell carcinoma by targeting GAB2
}

\author{
LIJUN MU $^{1 *}$, BING GUAN $^{1 *}$, JUANHUA TIAN ${ }^{1}$, XIANG LI $^{1}$, QINGZHI LONG ${ }^{1}$, MEIYU WANG $^{2}$, \\ WEN WANG ${ }^{3}$, JUNJUN SHE ${ }^{4}$, XUDONG LI ${ }^{1}$, DAPENG WU ${ }^{1}$ and YUEFENG DU ${ }^{1}$ \\ Departments of ${ }^{1}$ Urology, ${ }^{2}$ Imaging, ${ }^{3}$ Outpatient,${ }^{4}$ General Surgery, \\ The First Affiliated Hospital of Xi'an Jiaotong University, Xi'an, Shaanxi 710061, P.R. China
}

Received November 28, 2019; Accepted August 5, 2020

DOI: $10.3892 /$ or.2020.7759

\begin{abstract}
Renal cell carcinoma (RCC) is one of the most common malignant cancers in the adult urinary system worldwide. Tumor angiogenesis is a critical process during cancer progression, as it modulates carcinogenesis and metastasis. In recent years, microRNA-218 (miR-218) has been confirmed to play a crucial role in tumor suppression. However, the role of miR-218 in RCC angiogenesis remains unclear. In the present study, it was found that the expression of miR-218 was decreased in RCC tumor tissues and cell lines as detected by real-time PCR analysis. Tube formation assays and migration assays also confirmed that miR-218 inhibited the interaction between RCC cells and vascular endothelial cells by suppressing proangiogenic factor vascular endothelial growth factor A (VEGFA) in RCC cells. miR-218 also repressed the subcutaneous tumorigenesis of RCC cells in nude mice, and the corneal angiogenesis in rabbit eyes. The underlying molecular mechanism was elucidated; miR-218 targets GRB2-associated binding protein 2 (GAB2), thereby inhibiting the PI3K/AKT/mTOR/VEGFA pathway. These results provide new insights into the mechanism of RCC carcinogenesis and progression, suggesting that miRNA-218 may be a therapeutic target for the treatment of RCC.
\end{abstract}

\section{Introduction}

Renal cell carcinoma ( $\mathrm{RCC}$ ) is one of the most common malignant cancers in the adult urinary system worldwide (1). There are approximately 270,000 newly diagnosed and

Correspondence to: Dr Dapeng Wu or Dr Yuefeng Du, Department of Urology, The First Affiliated Hospital of Xi'an Jiaotong University, 277 Yanta West Road, Xi'an, Shaanxi 710061, P.R. China

E-mail: wudapeng0704@126.com

E-mail: duyuefeng113@163.com

*Contributed equally

Key words: renal cell carcinoma, microRNA 218, angiogenesis, GAB2, PI3K/AKT/mTOR approximately 116,000 deaths annually, worldwide (2), making it the second most malignant cancer of the urinary system, with a $40 \%$ mortality rate (3). Surgical resection is the best treatment for RCC. However, RCC is insidious, and approximately $30 \%$ of RCC patients have metastasis at the time of diagnosis, losing the chance of surgery (4). In addition, up to $40 \%$ of patients have local recurrence and/or distant metastasis (5). Unlike other urinary system tumors, $\mathrm{RCC}$ is resistant to radiotherapy and chemotherapy, leading to difficulties in the treatment of metastatic RCC (6). In recent years, molecular targeted drugs, as the first-line treatment of metastatic RCC, have prolonged patient survival. However, this is still merely effective for 6-15 months (7). Therefore, a more efficient therapeutic strategy for the metastasis of RCC and the underlying molecular mechanism of the initiation and development of RCC requires further exploration.

MicroRNAs (miRNAs/miRs) are a group of endogenous, short single-stranded non-coding RNA molecules containing approximately 18-25 nucleotides (8). miRNAs regulate the expression of targeted genes at the transcriptional or translational level mainly through binding to the $3^{\prime}$ untranslated regions (3'UTR) of targeted mRNAs, in order to regulate post-transcriptional expression of target genes (9). Furthermore, they are involved in cell growth, cell cycle control, apoptosis, tumor tissue infiltration and metastasis, angiogenesis and infinite proliferative potential (6). Tumor angiogenesis is a critical process during cancer progression, which modulates tumor growth and metastasis. Recent studies have reported that certain miRNAs may become involved in this procedure. Liang et al indicated that miR-153 suppresses the tube formation and the migration of endothelial cells by targeting angiopoietin 1 (ANG1) directly in breast cancer cells (10). miR-205 was found to significantly suppress angiogenesis and epithelial-mesenchymal transition (EMT) through the simultaneous targeting of vascular endothelial growth factor A (VEGFA), zinc finger e-box binding homeobox 1 (ZEB1), and downstream products in anaplastic thyroid carcinoma (11).

Studies have shown that microRNA-218 (miR-218) can inhibit the proliferation, migration, invasion and metastasis of cancer cells, which is a tumor suppressor in oral squamous cell carcinoma, gastric cancer, head and neck squamous cell carcinoma and other tumors (12-14). Small and Olson reported that 
the generation of the vascular system involves the regulation of miRNAs, in which miR-218 is an important regulator (15). Guan et al also confirmed that miR-218 regulates the angiogenesis of prostate cancer through the RPTOR-independent companion of MTOR complex 2 (RICTOR)/VEGFA axis, thereby inhibiting prostate cancer malignant progression (16). However, the role of miR-218 in RCC angiogenesis remains unclear.

In the present study, it was found that miR-218 could specifically bind to the target site of the 3'UTR of GRB2-associated binding protein 2 (GAB2), and negatively regulate its expression. By inhibiting the PI3K/AKT pathway, the expression of angiogenic factor VEGFA was suppressed. In addition, the interaction between renal carcinoma cells and vascular endothelial cells was disrupted, suppressing the proliferation, invasion, migration and angiogenesis of RCC. This result provides new insight into the mechanism of renal cell carcinogenesis and progression, suggesting that miRNA-218 may serve as a target for the treatment of RCC.

\section{Materials and methods}

Cell lines and cell culture. The human RCC cell lines ACHN, 769P and 7860 were obtained from the American Type Culture Collection (ATCC). Human umbilical vein endothelial cells (HUVECs), human kidney 2 (HK-2) and 293T cells were kindly provided by Dr Jer-Tsong Hsieh (University of Texas Southwestern Medical Center). The 293T cells and HUVECs were cultured in DMEM (Gibco; Thermo Fisher Scientific, Inc.) containing $10 \%$ fetal bovine serum (FBS) (Gibco; Thermo Fisher Scientific, Inc.). ACHN, 7860 and 769P cells were cultured in RPMI-1640 medium (Gibco; Thermo Fisher Scientific, Inc.) containing $10 \%$ FBS. All cells were cultured at $37^{\circ} \mathrm{C}$, under $5 \% \mathrm{CO}_{2}$ culture conditions.

Bioinformatics. Data for a total of 591 samples were downloaded from the TCGA website (https://portal.gdc.cancer. gov/). The downloaded data were the isoform expression quantification data of miRNA-Seq in the The Cancer Genome Atlas Kidney Renal Clear Cell Carcinoma (TCGA-KIRC) data collection. The mature miRNAs were calculated using the mature human miRNA annotation information (V21 version) from miRBase (http://www.mirbase.org/). The pathological parameters were statistically analyzed by Chi-square test, and the P-value of survival analysis was tested by $\log$ rank method. The Kaplan-Meier (KM) survival curve graph made by $\mathrm{R}$ package, and the expression level was distinguished by the median. The TargetScan (http://www.targetscan.org/) database was used to predict the targeted gene and binding site of miR-218, and GAB2 was selected.

Construction of stably miR-218-overexpressing and GAB2-knockdown cell lines. The lentivirus LV-miR-218 vector and the scrambled lentiviral vector LV-NC were constructed by GenePharma (Shanghai, China), which contained the green fluorescent protein (GFP) and anti-puromycin sequence. Lentiviral vectors that encoded short hairpin RNA (shRNA) targeting human GAB2 were also constructed by GenePharma. After these were verified through the DNA sequence, the lentivirus vectors were used to infect the ACHN, 7860 and 769P cell lines. Then, $2 \times 10^{6} \mathrm{RCC}$ cells were seeded in $6-\mathrm{cm}$ dishes. Afterwards, the medium was replaced after the cells were adhered to the wall, and $20-\mu 1$ lentivirus vectors $\left(1 \times 10^{8} \mathrm{TU} / \mathrm{ml}\right)$ were added to the medium according to the optimal multiplicity of infection (MOI) value of 10 , along with $8 \mu \mathrm{g} / \mathrm{ml}$ of polybrene. The medium was changed after $48 \mathrm{~h}$, and $2 \mu \mathrm{g} / \mathrm{ml}$ of puromycin was added to select and maintain the stably miR-218-overexpressing and GAB2-knockdown RCC cell lines.

Total RNA and microRNA isolation. RCC cells were harvested when $50 \%$ confluence was reached. The isolation of cellular total RNA and microRNA was separately performed using a total RNA isolation kit (Feijie; RNAfast200) and a miRcute miRNA isolation kit (Tiangen; DP501) according to manufacturer's protocol.

$R N A$ reverse transcription. The reverse transcription of total RNA and microRNA was separately preformed using a PrimeScript $^{\mathrm{TM}}$ RT reagent kit (Takara; RR037A) and miRcute Plus miRNA First-Strand cDNA Synthesis Kit (Tiangen; KR211), according to the manufacturer's instructions.

Analysis of gene expression. The transcriptional expression of the target genes was detected using SYBR Premix Ex Taq II (Takara; RR820A) using the CFX96 Real-time PCR system (Bio-Rad Laboratories, Inc.). The PCR reaction mixtures were incubated at $95^{\circ} \mathrm{C}$ for $30 \mathrm{sec}$ for initial denaturation; followed by 40 cycles at $95^{\circ} \mathrm{C}$ for $5 \mathrm{sec}, 60^{\circ} \mathrm{C}$ for $30 \mathrm{sec}$. The relative gene expression was calculated using the $2^{-\Delta \Delta \mathrm{Cq}}$ method (17). The primers used for PCR are provided in Table SI.

The protein expression of target genes was analyzed by western blot analysis. The RCC cells were gently washed three times using pre-cooled PBS until $80 \%$ confluence was reached, and lysed by RIPA containing $2 \%$ protease inhibitor PMSF. Approximately $30 \mu \mathrm{g}$ of each protein sample was added to $10-12 \%$ SDS-PAGE. Then, these proteins were separated and blotted to polyvinylidene fluoride membranes. Afterwards, the membranes were blocked with 5\% skim milk for $1 \mathrm{~h}$ at room temperature, and incubated with primary antibodies against GAB2 (1:500, product code ab32365; Abcam), protein kinase B (AKT) (1:500; product code ab8805; Abcam), phosphorylated (p)Akt (1:2,500; product code ab81283; Abcam), mTOR (1:1,000, product code ab2732; Abcam), pmTOR (1:1,000; product code ab109268; Abcam), hypoxia inducible factor 1 subunit $\alpha$ (HIF1 $\alpha$ ) (Abcam; [H1alpha67] (ab1), VEGFA (1:200; product code ab1316; Abcam) and glyceraldehyde 3-phosphate dehydrogenase (GAPDH) (1:1,000; cat. no. SS12002; Kangcheng) at $4^{\circ} \mathrm{C}$ overnight. Afterwards, these membranes were washed with TBST before incubation with horseradish peroxidase-conjugated secondary antibodies (1:2,000, cw0102 and cw0103; $\mathrm{CWBIO}$ ) for $1 \mathrm{~h}$ at room temperature. Finally, the protein bands were subjected to the ChemiDoc XRS Visualize System (Bio-Rad Laboratories) for visualization and image collection.

Luciferase reporter assay. In order to determine whether miR-218 directly targets the 3'UTR of GAB2, two types of reporter plasmids were constructed using the GP-miRGLO vector (GenePharma): Wild-type GAB2 3'UTR reporter 
plasmid (WT) and mutated GAB2 3'UTR reporter plasmid (MUT). A total of $8 \times 10^{4} 293 \mathrm{~T}$ cells were seeded in a 24-well plate, and co-transfected with $50 \mathrm{nM}$ of miR-218 or NC mimic, and the luciferase reporter plasmid WT or MUT. At $48 \mathrm{~h}$ after transfection, the luciferase activity was measured using a Dual Luciferase Assay kit (Promega Corp.), according to the manufacturer's instructions.

Conditioned medium collection. A total of $8 \times 10^{5}$ RCC cells were seeded in a 6-cm culture dish. After adhesion, these cells were gently washed three times with serum-free medium (SFM), fed $4 \mathrm{ml}$ of SFM, and cultured for $24 \mathrm{~h}$. Then, the supernatant was centrifuged and collected as conditioned medium (CM).

In vitro HUVEC migration assays. A total of $4 \times 10^{4} \mathrm{HUVECs}$ in SFM were placed into the upper chamber of a 24-well plate, while the lower chamber was filled with $1 \mathrm{ml}$ of different CMs, or seeded with $1 \times 10^{4}$ RCC cells in SFM. After $16 \mathrm{~h}$ of incubation, the migrated cells were fixed with $4 \%$ paraformaldehyde and stained with $0.1 \%$ crystal violet at room temperature for $10 \mathrm{~min}$. The number of migrated cells was counted by upright microscopic imaging system (Olympus, Japan) in six random fields per well (x200 magnification).

HUVEC tube formation assays. A total of $1 \times 10^{5}$ HUVECs mixed with CM or SFM were seeded in a Matrigel-coated 24-well plate per well. After incubation for $4 \mathrm{~h}$, tube-like vascular structures were observed by inverted microscope (Olympus, Japan). Merely perfectly continuous tubes between two branching points were considered as a tube.

ELISA assay. The secreted VEGFA in CMs was assessed using a RayBio ${ }^{\circledR}$ Human VEGF ELISA kit (RayBiotech Inc.), according to the manufacturer's instructions.

CCK- 8 assay. CCK- 8 assay was performed to mesure the growth rate of cells using Cell Counting Kit-8 (Selleck Chemicals), according to the manufacturer's instructions.

In vivo studies. A total of 8 male New Zealand white rabbits, three months old, weighing approximately $2 \mathrm{~kg}$, and 10 male mice, six weeks old, weighing about $20 \mathrm{~g}$, approximately used in the present study and obtained from Xi'an Jiaotong University Animal Eperimental Center. Housing condition consisted of $23^{\circ} \mathrm{C}, 15 \mathrm{~Pa}$ higher than room air pressure and 12-h light/dark cycle. All procedures for animal experiments were performed in accordance to the Guidelines of the Institutional Animal Care and Use Committee of Xi'an Jiaotong University (18). In order to develop the xenograft tumor models, $2 \times 10^{6}$ cells were subcutaneously injected into both sides of the plank region of nude mice, with five mice in each group. The tumor size was measured once a week, and the tumor volume $(\mathrm{V})$ was calculated by the formula $\mathrm{V}=\left(\right.$ length $\mathrm{x}$ width $\left.{ }^{2}\right) / 2$. After four weeks, the mice were euthanized by cervical dislocation, and the tumors were harvested and weighed, and then fixed with $4 \%$ paraformaldehyde. For the rabbit cornea assays, six male New Zealand white rabbits were randomly divided into two groups. Pentobarbital sodium $(30 \mathrm{mg} / \mathrm{kg}$ ) was injected into the auricular vein of rabbits, and then tetracaine eye drops were used for local anesthesia. After that, $1 \times 10^{5}$ cells were injected into the surgically produced micro pocket $(1.5 \times 3.0 \mathrm{~mm})$ in the 6 and 12 o'clock points of the right eye. After four weeks, the cornea angiogenic responses were observed. When the observation was completed, rabbits were euthanized by injection of pentobarbital sodium $(100 \mathrm{mg} / \mathrm{kg})$. The animal experiments conducted in the present study were approved by the Ethics Committee of The First Affiliated Hospital of Xi'an Jiaotong University (2013065).

Immunohistochemistry. The nude mouse xenograft specimens were analyzed by immunohistochemistry assay (IHC) using the EnVision ${ }^{\mathrm{TM}}$ System (Dako), according to the manufacturer's instructions. The primary antibodies were GAB2 (Proteintech, 22549-1-AP, dilution 1:50), VEGFA (Abcam, ab1316, dilution 1:50), proliferating cell nuclear antigen (PCNA) (Proteintech, 10205-2-AP, dilution 1:200), and CD31 (Abcam, ab28364, dilution 1:50). According to the intensity of the nuclear or cytoplasmic staining, the average intensity score of the positive cells was divided into four levels: None, 0; weak, 1; intermediate, 2 and strong, 3. According to the percentage of positively stained cells, the score was divided into four grades: $0-25=1 ;>25-50=2 ;>50-75=3$, and $>75-100 \%=4$. These two scores were multiplied to obtain the total staining score, which ranged from 0 to 12 . Three fields were randomly selected for the examination.

Statistical analysis. For the differences between two groups (Student's t-test) and multiple groups (one-way ANOVA followed by Dunnett's multiple comparison tests), Pearson's correlation and linear regression were analyzed using the GraphPad Prism version 6.0 software (GraphPad Software, Inc.). A P-value of $<0.05$ was considered as indicative of statistical significance.

\section{Results}

Expression of miR-218 in renal cell carcinoma. The expression of miR-218 in human RCC tissue samples and adjacent normal tissues was analyzed using the TCGA database. The results found that miR-218 expression was significantly lower in the tissue samples when compared to that in the adjacent normal tissues (Fig. 1A). In addition, real-time PCR results also revealed a significantly higher miR-218 expression in human renal cortical proximal tubular epithelial HK-2 cells, when compared to the RCC ACHN, 7860 and 769P cell lines (Fig. 1B). Intriguingly, the overall survival rate of RCC samples with high expression of miR-218 was lower than the overall survival rate of the RCC samples with low expression (Fig. S1), and the expression of miR-218 was higher in the more malignant stages and grades (Table SII).

miR-218 inhibits RCC angiogenesis in vitro. Three different RCC cell lines stably overexpressing miR-218 (LV-miR-218) were established (Fig. 1C-E). Endothelial cell recruitment assay was performed to explore the effect of miR-218 on the migration of HUVECs. Under the condition of conditioned medium (CM) or HUVEC co-culture, the migration ability of the HUVECs were significantly restrained by overexpression of miR-218 (Fig. 2A and B). Furthermore, CCK-8 assay 

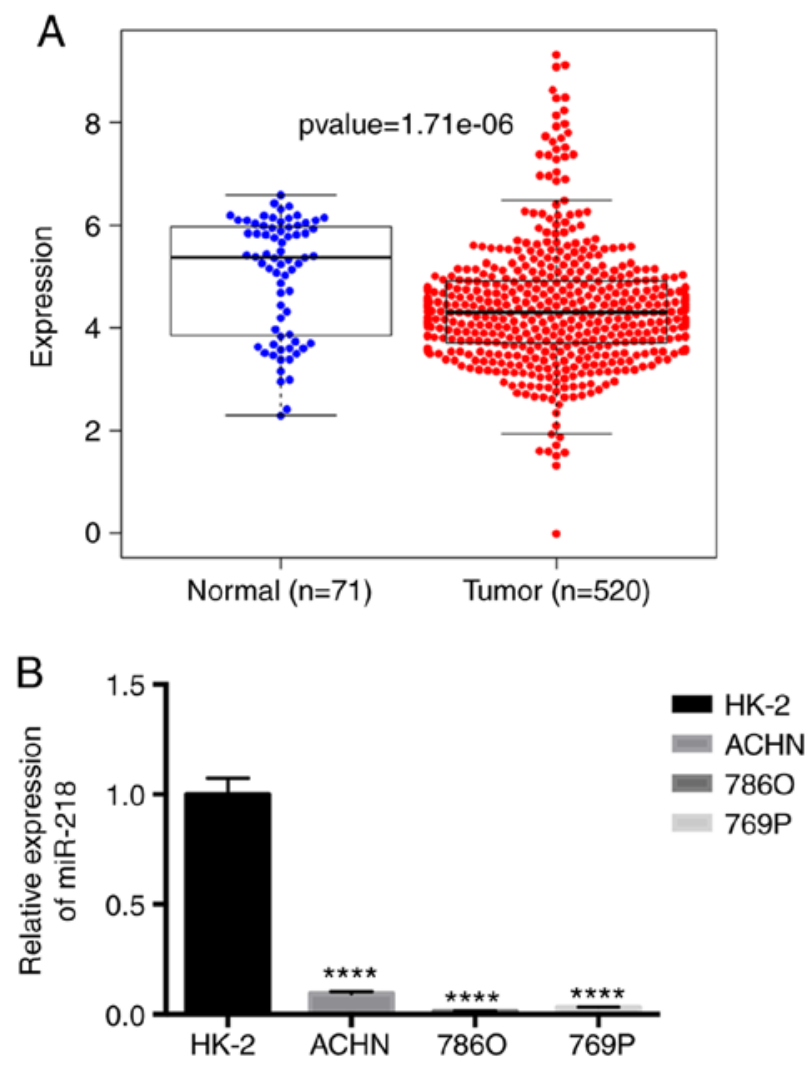

C

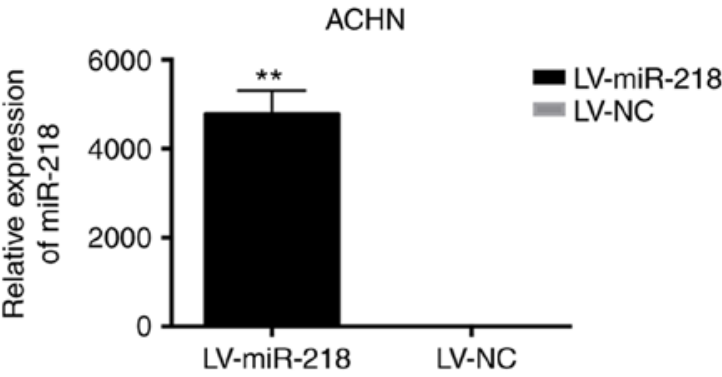

D

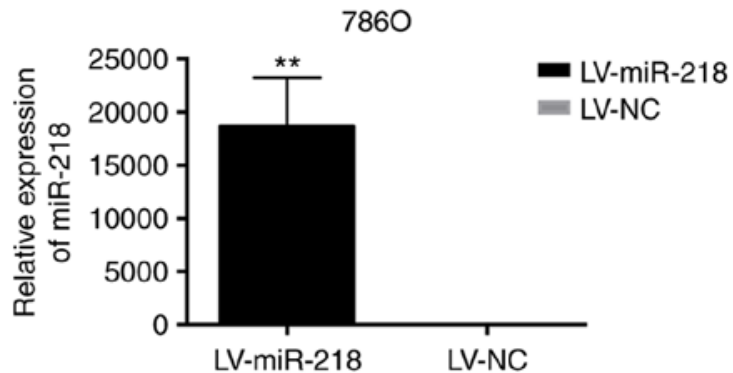

$\mathrm{E}$

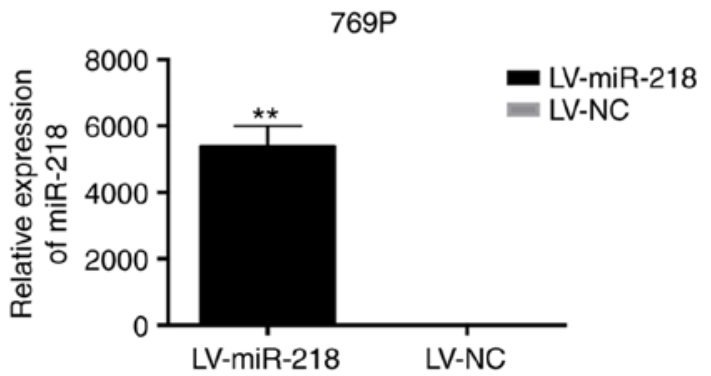

Figure 1. miR-218 is downregulated in RCC tissues and cells. (A) miR-218 expression level in human RCC tissues was compared between normal adjacent tissues and tumor tissues by analyzing the data downloaded from the TCGA database. (B) Expression level of miR-218 was analyzed by miRNA real-time PCR in a normal renal cortical proximal tubular epithelial cell line HK-2 and RCC cell lines (ACHN, 786O and 769P). ${ }^{* * * *} \mathrm{P}<0.0001$ compared with the HK-2 cells. (C-E) Stable miR-218-overexpressing RCC cell lines were constructed. ${ }^{* *} \mathrm{P}<0.01$ compared to the LV-NC group. These data are representative of three independent experiments. The asterisks indicate a significant difference. RCC, renal cell carcinoma; TCGA, The Cancer Genome Atlas.

was conducted to evaluate the effect of miR-218 on HUVEC proliferation. Compared to the LV-NC group, the CM of miR-218-overexpressing ACHN cells inhibited the proliferation of HUVECs (Fig. 2C-E). The tube formation assay also revealed that HUVECs formed less tube-like structures in miR-218-overexpressing cells compared to the LV-NC group, indicating that miR-218 could inhibit the angiogenesis of RCC (Fig. 2F). In addition, the expression of VEGFA, which is the most important angiogenic factor in tumor angiogenesis, was decreased in RCC cells with miR-218 overexpression, when compared to the LV-NC group (Fig. 2G and H). The ELISA assay results revealed that the concentration of VEGFA protein secreted by miR-218-overexpressing RCC cells was also decreased (Fig. 2I), illustrating that miR-218 inhibited the secretion of VEGFA in RCC cells. In addition, the mRNA expression of EMT-related markers E-cadherin and vimentin was investigated, and the expression of vimentin was significantly decreased while E-cadherin expression was significantly increased in the LV-miR-218 group. The results indicated that the overexpression of miR-218 induced a slight migration and invasion change (Fig. S2, left panel).

miR-218 targets GAB2 by binding to 3 '-UTR. In order to find the binding site of miR-218, we searched the TargetScan database and selected GAB2 (Fig. 3A). Then, a dual luciferase reporter gene assay was performed. The wild-type (WT) and mutant (MUT) sequences of the GAB2 3'UTR were cloned into the reporter plasmids (Fig. 3A). Overexpression of miR-218 was able to repress the luciferase activity (55\%) of the wild-type (WT) reporter, but not the mutant (MUT) reporter (Fig. 3B), indicating that miR-218 is capable of specifically targeting the 3'UTR of GAB2. Furthermore, the expression of GAB2 in RCC cell lines at both the mRNA and protein level was indeed significantly suppressed by the overexpression of miR-218 (Fig. 3C and D), when compared to the LV-NC group.

GAB2 plays a crucial role in RCC angiogenesis. In order to further investigate the mechanism of miR-218 in regulating RCC angiogenesis by targeting GAB2, GAB2-knockdown RCC cell lines were constructed using ACHN and 7860 cells, and the knockdown efficiency was confirmed at both the mRNA and protein levels (Fig. 4A and B). Consistently, after downregulating GAB2, the migration (Fig. 4C and D) and tube formation (Fig. 4E) ability of HUVECs were significantly inhibited, and the EMT of RCC cells was also slightly suppressed (Fig. S2, right panel). In addition, western blot analysis (Fig. 4B) and ELISA (Fig. 4F) assays revealed a significant decrease in VEGFA protein expression and 
A

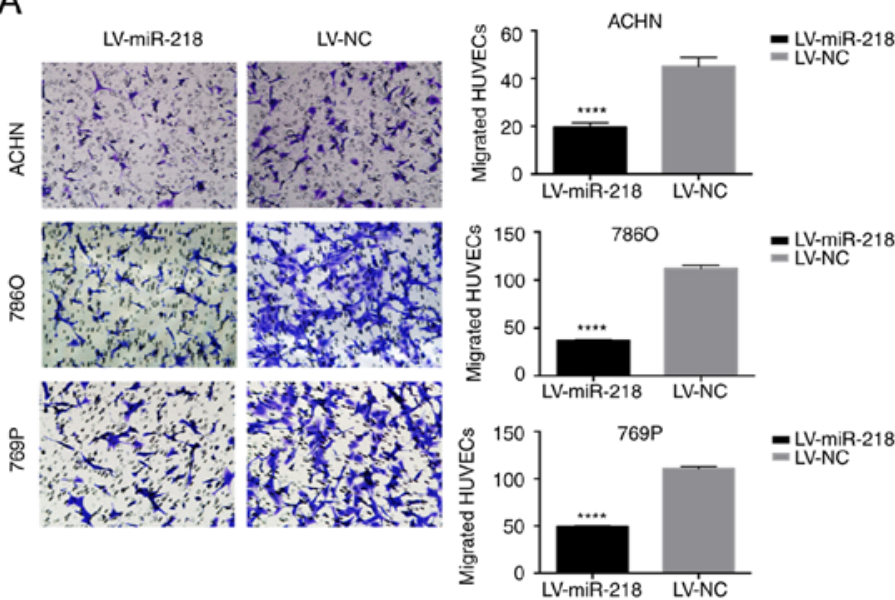

C

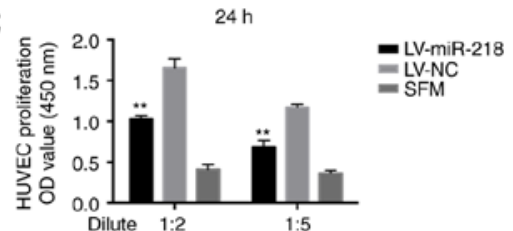

B
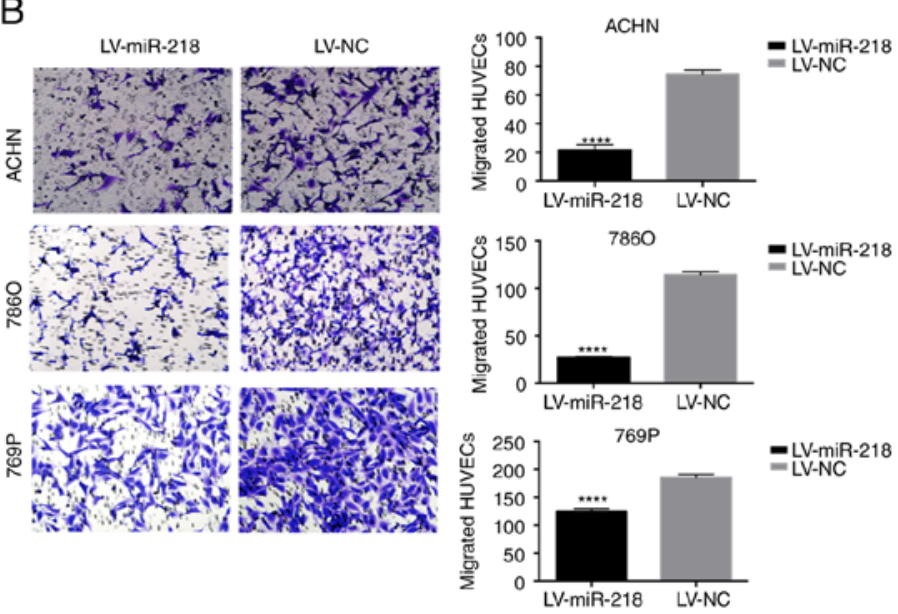

E
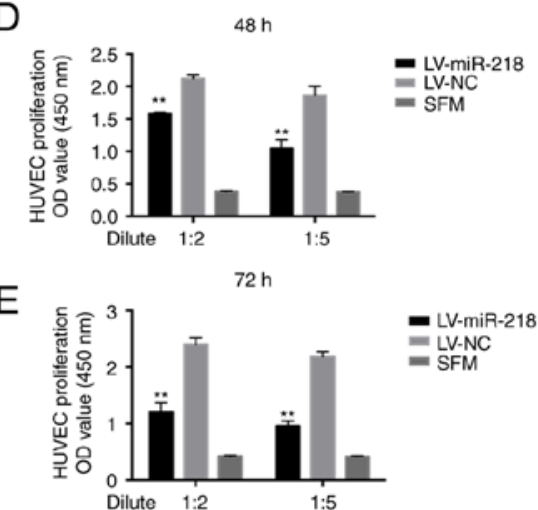

F
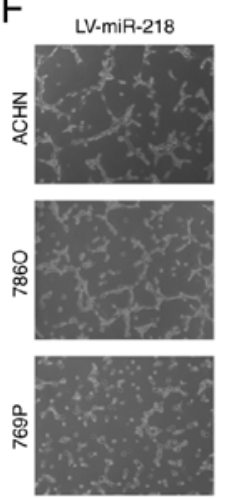

LV-NC
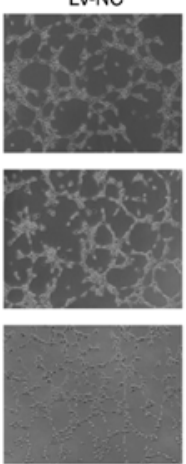
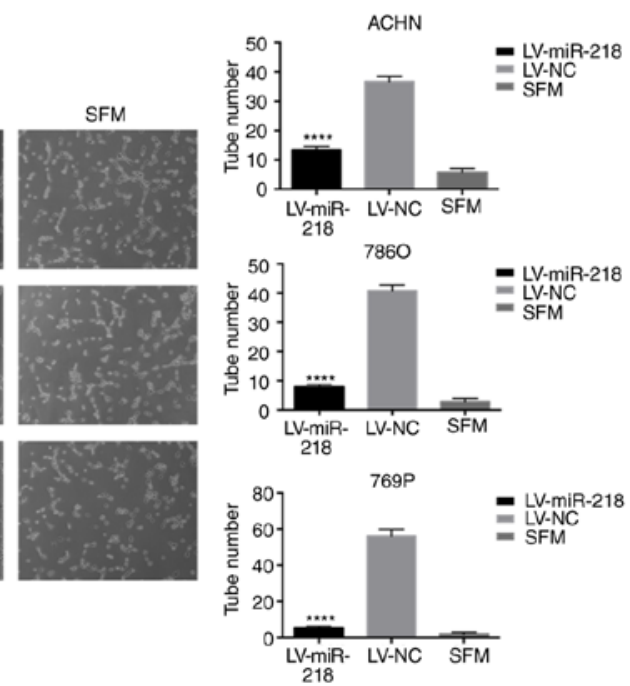

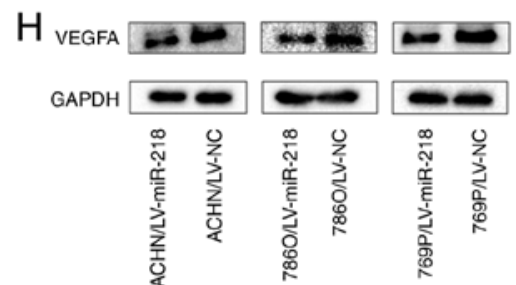

$=\stackrel{\operatorname{LV}-\mathrm{miR}-218}{\mathrm{LV}-\mathrm{NC}} \mathrm{G}$
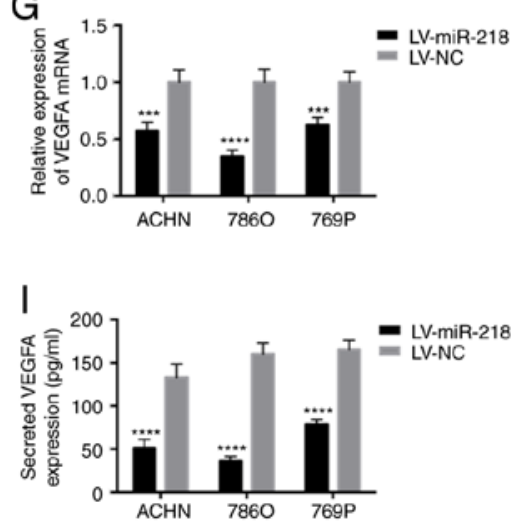

Figure 2. miR-218 inhibits HUVEC migration, proliferation and tube formation in vitro, and inhibits VEGFA expression. (A) Overexpression of miR-218 suppresses the recruitment of HUVECs in a co-cultured system. Cancer cells cultured in the bottom of a 24-well plate were used to recruit HUVEC seeding on the upper chamber of Transwell insets. ${ }^{* * * *} \mathrm{P}<0.0001$ compared with the LV-NC group. (B) Overexpression of miR-218 decreased the recruitment of HUVECs through the conditioned medium (CM), which was collected from the ACHN, 786 O and 769P/LV-miR-218 or LV-NC cells. HUVECs were seeded on the upper chamber of the Transwell insets within $16 \mathrm{~h}$. The migrated cells in six random fields per well were counted (magnification, $\mathrm{x} 200$ ). ${ }^{* * * *} \mathrm{P}<0.0001 \mathrm{compared}$ with the LV-NC group. (C-E) miR-218 overexpression reduced the proliferation of HUVECs. HUVECs were treated with serum-free medium (SFM) or diluted CMs for 24,48 and $72 \mathrm{~h}$ before the CCK- 8 assay. ${ }^{* *} \mathrm{P}<0.01$ compared with the LV-NC group. (F) miR-218 overexpression suppressed the tube formation of HUVECs. HUVECs diluted in SFM or CMs were added into Matrigel-coated wells and incubated for $4 \mathrm{~h}$. The representative images of tube-like structures were captured (left), and the tube number in the whole field was counted (magnification, x100) (right). **** $\mathrm{P}<0.0001$ compared with the LV-NC group. (G) Real-time PCR was used to analyze the expression level of VEGFA in ACHN, 7860 and 769P cells transfected with LV-NC or LV-miR-218. ${ }^{* * *} \mathrm{P}<0.001$ and ${ }^{* * * *} \mathrm{P}<0.0001$ compared to the LV-NC group. (H) The VEGFA protein level was analyzed by western blot analysis. (I) The concentration of secreted VEGFA protein in the CMs was determined by ELISA. ${ }^{* * * *} \mathrm{P}<0.0001$ compared to the LV-NC group. These data are representative of three independent experiments. HUVECs, human umbilical vein endothelial cells; VEGFA, vascular endothelial growth factor A. 
A

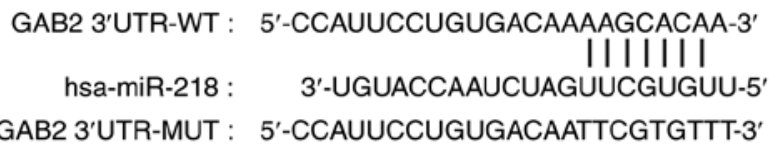

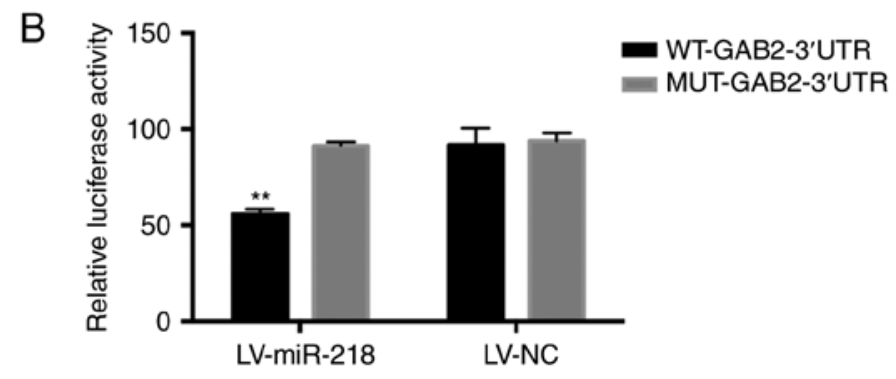

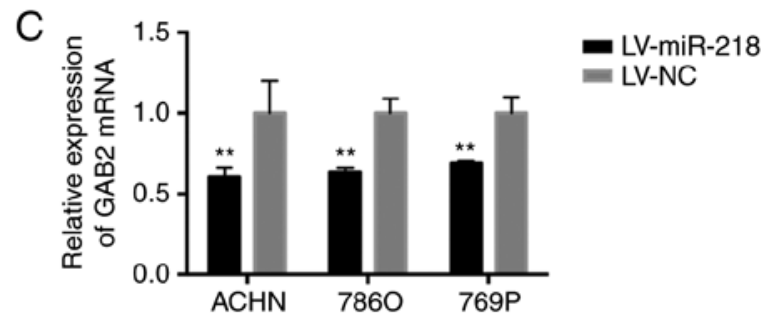

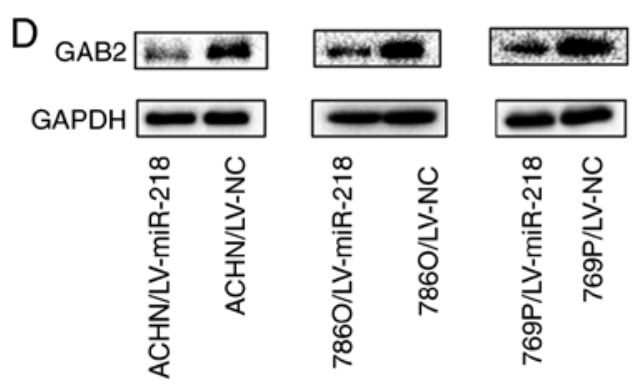

Figure 3. miR-218 targets GAB2 by binding to its 3'UTR. (A) The GAB2 3'UTR regions containing the wild-type (WT) or mutant (MUT) binding site and the sequence complementarity between miR-218 and the GAB2 3'UTR are shown. (B) The relative luciferase activity was analyzed after transfection with the WT or MUT 3'UTR reporter plasmids in LV-NC or LV-miR-218 293T cells. Overexpression of miR-218 suppressed the luciferase activity of the wild-type reporter (55\%). ${ }^{* *} \mathrm{P}<0.01$ compared to the MUT reporter. (C) Real-time PCR was used to analyze the expression level of GAB2 in ACHN, 786O and 769P cells transfected with LV-NC or LV-miR-218. ${ }^{* *} \mathrm{P}<0.01$ compared to the LV-NC group. (D) GAB2 protein level was analyzed by western blot analysis. The results are representative of three independent experiments. The values were presented as the mean \pm standard deviation (SD). GAB2, GRB2-associated binding protein 2; UTR, untranslated region.

secretion in the shGAB2 group when compared with the shNC group.

miR-218 regulates the PI3K/AKT/mTOR axis by targeting $G A B 2$. Overexpression of miR-218 in RCC cells not only reduced $\mathrm{GAB} 2$ expression, but also inhibited the phosphorylation of AKT (p-AKT) at position 473, the phosphorylation of mTOR (p-mTOR), and the protein expression of HIF-1 $\alpha$ (Figs. 5A and S3). Consistently, knockdown of GAB2 in RCC cells exhibited similar results (Fig. 5B), indicating that miR-218 exerts its role on inhibition of RCC angiogenesis through the GAB2/PI3K/AKT/mTOR/HIF-1 $\alpha /$ VEGFA axis.

miR-218 inhibits RCC tumor angiogenesis in vivo. In order to investigate the effect of miR-218 on the tumorigenicity of RCC cells in vivo, miR-218-overexpressing (LV-miR-218)/control (LV-NC) 7860 cells were subcutaneously injected into the flanks of nude mice. The tumor growth was slower in the 786O/LV-miR-218 group, showing significantly smaller tumor size and lower tumor weight, when compared to the control group (Figs. 6A and B and S4). In addition, the immunohistochemistry (IHC) staining exhibited fewer CD31 (endothelial cell marker)-positive tissues in the 7860/LV-miR-218 tumors, when compared to the LV-NC group. Similar results were also observed in the PCNA (proliferating cell nuclear antigen), GAB2 and VEGFA staining in these tumor tissues (Fig. 6C-F).

The rabbit cornea angiogenesis assay is a more specific animal model to evaluate tumor angiogenesis. Therefore, a tumorigenic test in the cornea of rabbit eyes was performed using 7860/LV-miR-218 and 7860/LV-NC cells. At four weeks after tumor transplantation, 786O/LV-NC cells induced a neovascular response and visible tumors in the cornea. However, 7860/LV3-miR-218 cells lost this ability (Fig. 6G). Overall, this demonstrated that miR-218 not only regulates
RCC tumorigenicity, but also plays an important role in suppressing RCC angiogenesis.

\section{Discussion}

With the deepening investigation on microRNAs (miRNAs/miRs), the basic characteristics and biological functions of miRNAs in regards to development have been recognized. As for miR-218, studies have confirmed that it can inhibit cancer cell proliferation, migration, invasion and metastasis, and that it is a tumor suppressor in prostate cancer, oral squamous cell carcinoma, lung cancer, gastric cancer, head and neck squamous cell carcinoma, and several other tumors $(12-14,16)$. There is an obvious inhibition or mutation of miR-218 expression in tumors, which may be correlated to the hypermethylation/demethylation of $\mathrm{CpG}$ islands around the miRNA promoter and histone $\mathrm{H} 3$ acetylation (19). Leite et al (20) reported that metastatic prostate cancer presents with sharp decreases in miR-218, when compared to high grade prostate intraepithelial neoplasia (HGPIN) and localized prostate cancer, indicating the expression change of miR-218 involved in the progression of metastatic prostate cancer, and that angiogenesis is one of the key steps in this process. Researchers have used HITS-CLIP technology to demonstrate that miR-218 targets multiple functional genes, including nuclear factor $-\kappa \mathrm{B}$ subunit $1(\mathrm{NF}-\kappa \mathrm{B})$, roundabout guidance receptor 1 (ROBO1), LIM and SH3 protein 1 (LASP1), paxillin (PXN), cyclin-dependent kinase 6 (CDK6) and cathepsin B (CTSB), and regulates multiple signaling pathways, including cell proliferation, circulation, metabolism and cell viability $(21,22)$. In the present study, it was demonstrated that miR-218 is lowly expressed in RCC tissues and cells, and that RCC samples with high expression of miR-218 presented with extended overall survival, suggesting that the loss of 
A

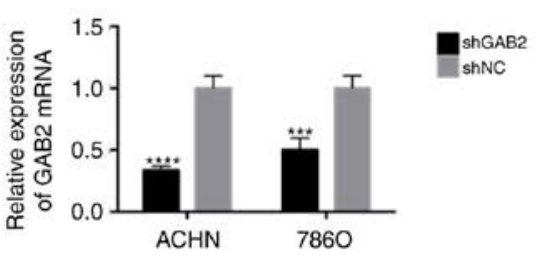

C

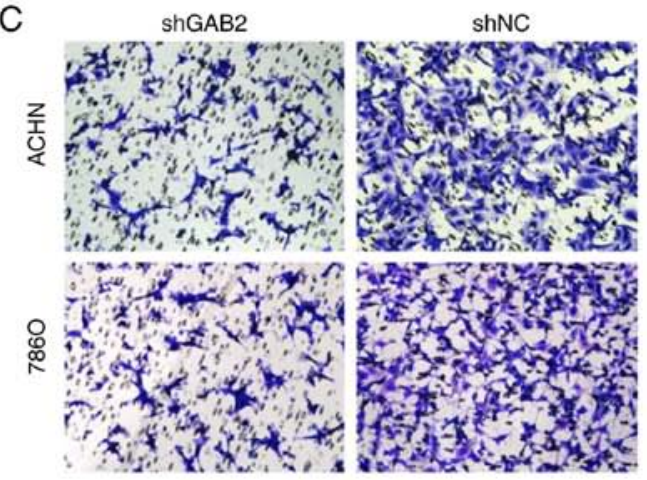

D

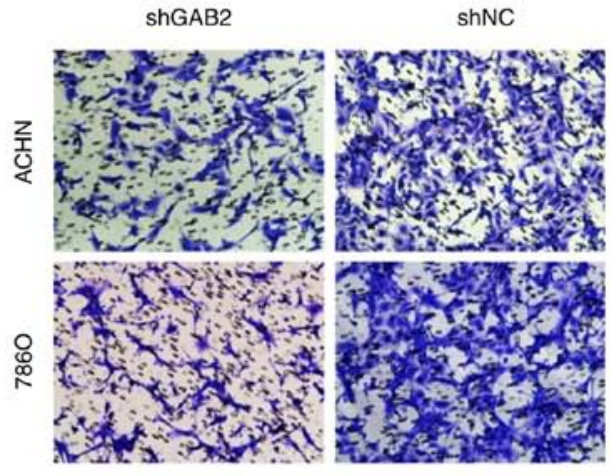

B GAB2

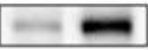

VEGFA

GAPDH
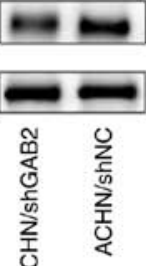

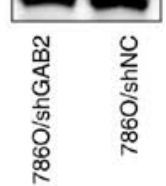

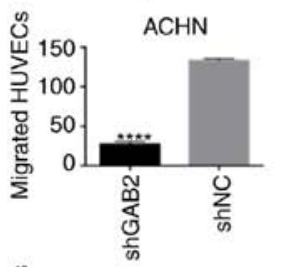

mhGAB2
shNC

shNC

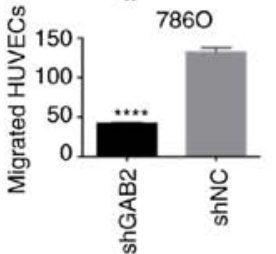

shGAB2

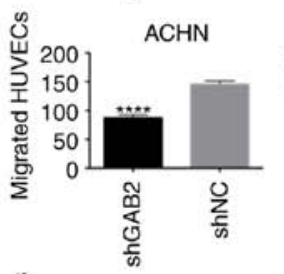

o 7860

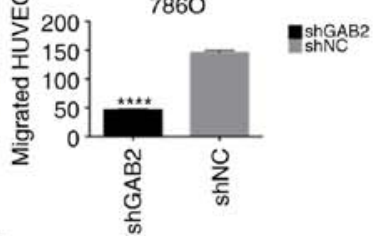

E
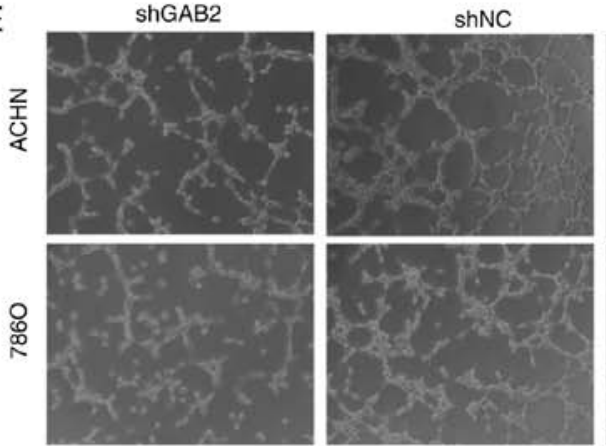

$\mathrm{F}$

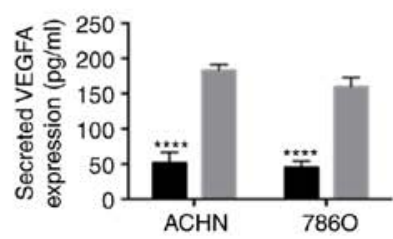

SFM
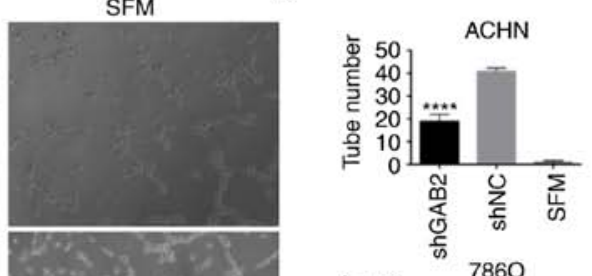

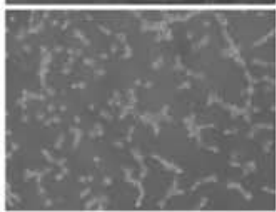

shGAB

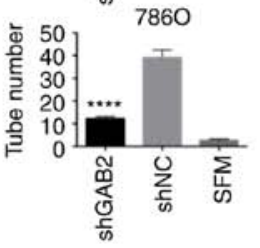

shGAB2
shNC

mshGAB2
shNC

$\lim _{\substack{x \rightarrow \infty \\ s \in \rightarrow \infty}}$

Figure 4. GAB2 plays a crucial role in RCC angiogenesis. (A and B) Real-time PCR and western blot analysis were used to confirm the knockdown of GAB2 in ACHN and 7860 cells transfected with LV-shGAB2 both at the mRNA and protein level. GAB2 knockdown decreased VEGFA at the protein level. ${ }_{* * * *} \mathrm{P}<0.001$ and ${ }^{* * * *} \mathrm{P}<0.0001$ compared to the shNC group. (C) GAB2 knockdown decreased the recruitment of HUVECs in a co-cultured system. Cancer cells that were cultured in the bottom of a 24 -well plate were used to recruit HUVECs. ${ }^{* * * *} \mathrm{P}<0.0001$ compared to the shNC group. (D) GAB2 knockdown decreased the recruitment of HUVECs through the conditioned medium (CM) collected from the ACHN, 786O/LV-shGAB2, and ACHN, 786O/LV-shNC cells. The migrated cells in six random fields per well were counted (magnification, $\mathrm{x} 200$ ). ${ }^{* * * *} \mathrm{P}<0.0001$ compared to the shNC group. (E) GAB2 knockdown reduced the tube formation of HUVECs diluted in SFM or CMs. The representative images of tube-like structures are shown, and the tube numbers in the whole field were counted (magnification, $\mathrm{x} 100)$ ). ${ }^{* * * * *} \mathrm{P}<0.0001$ compared to the shNC group. $(\mathrm{F})$ The concentration of secreted VEGFA protein in the CMs was determined by ELISA. The values were presented as the mean \pm standard deviation (SD). GAB2, GRB2-associated binding protein 2; RCC, renal cell carcinoma; HUVECs, human umbilical vein endothelial cells; SFM, serum-free medium; CM, conditioned medium; VEGFA, vascular endothelial growth factor A. 
A
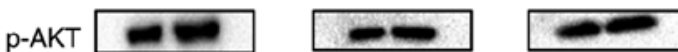

p-mTOR
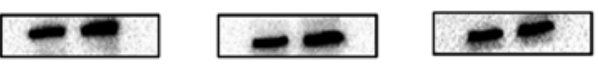

$\mathrm{HIF}-1 \alpha$
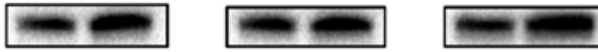

GAPDH
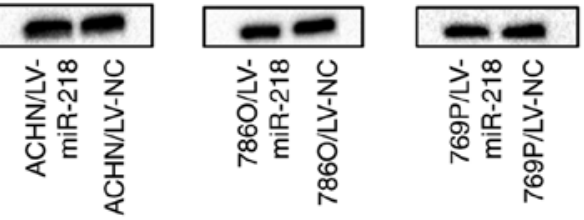

B
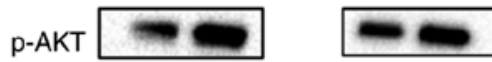

p-mTOR
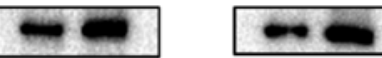

HIF-1 $\alpha$
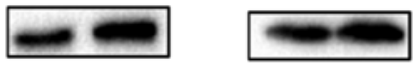

GAPDH

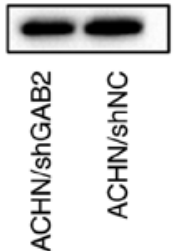

\begin{tabular}{|c|c|}
\hline 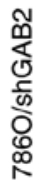 & $\begin{array}{l}0 \\
Z \\
\frac{1}{\infty} \\
0 \\
0 \\
\mathbb{N}\end{array}$ \\
\hline
\end{tabular}

Figure 5. miR-218 regulates the PI3K/AKT/mTOR signaling pathway by targeting GAB2. (A) The protein level of phosphorylated (p)-AKT at Ser-473, p-mTOR and HIF1 $\alpha$ were analyzed in miR-218-overexpressing RCC cells by western blot analysis. GAPDH was used as an internal control. (B) The protein level of p-AKT at Ser-473, p-mTOR, HIF1 $\alpha$, and VEGFA were analyzed in GAB2-knockdown RCC cells by western blot analysis. GAPDH was used as an internal control. These data are representative of three independent experiments. GAB2, GRB2-associated binding protein 2; RCC, renal cell carcinoma; mTOR, mammalian target of rapamycin; HIF1 $\alpha$, hypoxia inducible factor 1 subunit $\alpha$; VEGFA, vascular endothelial growth factor A.

A

7860/LV-miR-NC

7860/LV-miR-218

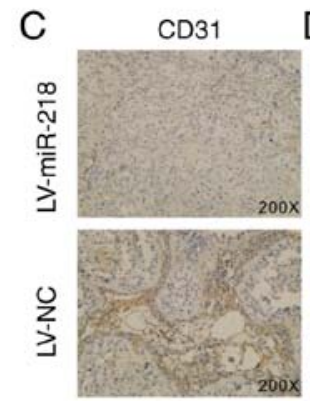

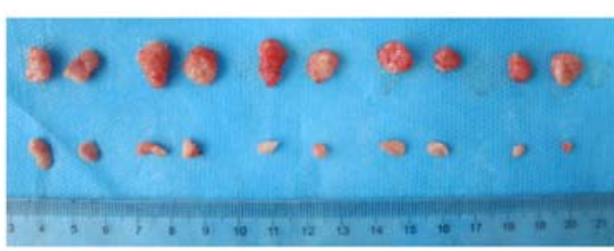

B

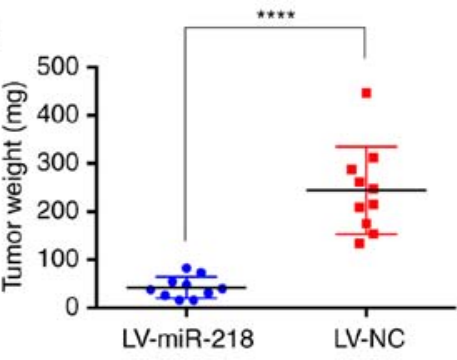

Figure 6. miR-218 inhibits tumor growth and angiogenesis in vivo. (A) Subcutaneous xenografts of 786O/LV-miR-218 and 786O/LV-NC subclones were harvested at four weeks after inoculation. (B) The tumor weights between the two groups. The values are presented as mean \pm standard deviation (SD). ${ }_{* * * * *} \mathrm{P}<0.0001$. (C-F) Expression of CD31, GAB2, PCNA and VEGFA were analyzed in paraffin-fixed tumor sections from 786O/LV-miR-218 and 786O/LV-NC xenografts by immunohistochemistry. Representative images are shown at magnification x200 (CD31) or x400 (GAB2, PCNA and VEGFA). (G) The $786 \mathrm{O} / \mathrm{LV}-\mathrm{NC}$ cells, but not the $786 \mathrm{O} / \mathrm{LV}$-miR-218 cells, induced the angiogenesis in rabbit cornea $(\mathrm{n}=3)$. Neonatal vessels invaded the cornea, connecting the tumor (yellow arrow) and the cornea limbal vascular plexus. GAB2, GRB2-associated binding protein 2; RCC, renal cell carcinoma; PCNA, proliferating cell nuclear antigen; VEGFA, vascular endothelial growth factor A.

miR-218 may play a key regulatory role in the development of RCC. In addition to the high miR-218 expression at lower ages, intriguingly, in the TNM staging and grading of RCC, miR-218 presented a reverse tendency: Higher in the more malignant phases. Moreover, the overall survival rate of RCC samples also shown an opposite trend: Lower miR-218 expression in higher overall survival rate, which probably means that the miR-218-related regulation is complex, and that its clinical role needs to be explored from multiple aspects (Table SII).
It is known that one main mechanism of RCC metastasis is tumor angiogenesis, which is an essential prerequisite for tumor growth and metastasis. As early as 1971, Folkman first proposed that tumor growth depends on angiogenesis (23). RCC has been widely accepted as a vascular-rich tumor (24). In the early stage of tumorigenesis, the tumor grows slowly due to the lack of a neovascular system. However, as tumor-secreted factors increase, the neovasculature begins to form. Thus, the tumor rapidly grows. Without the support of neovascularization, these tumors would 


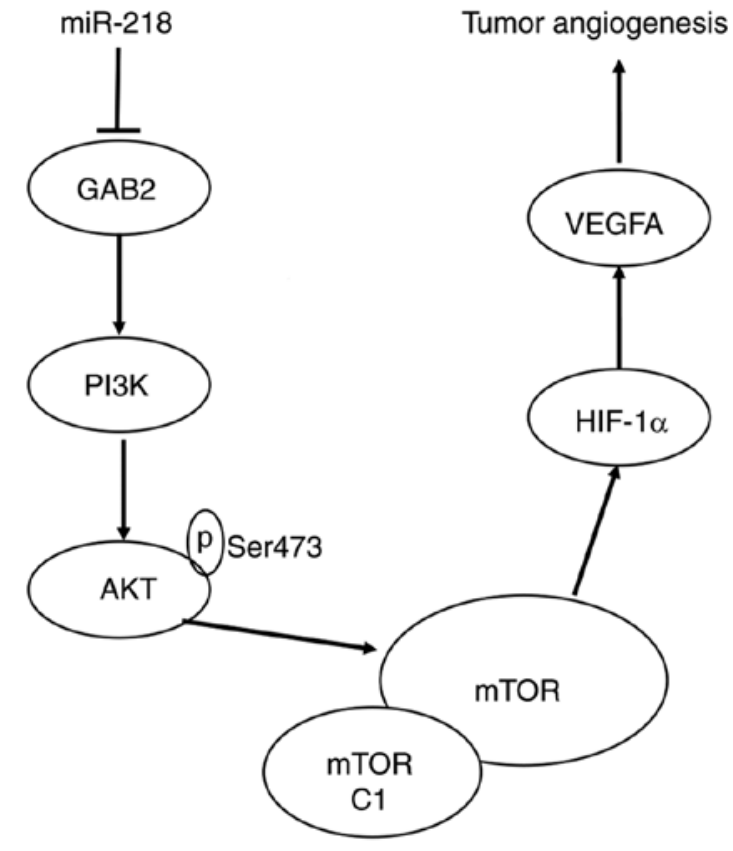

Figure 7. Schematic representation of the roles of miR-218 on the GAB2/PI3K/AKT/mTOR/HIF-1 $\alpha /$ VEGFA pathway in renal cell carcinoma angiogenesis. GAB2, GRB2-associated binding protein 2; RCC, renal cell carcinoma; mTOR, mammalian target of rapamycin; HIF1 $\alpha$, hypoxia inducible factor 1 subunit $\alpha$; VEGFA, vascular endothelial growth factor A; PI3K, phosphoinositide 3-kinase.

not exceed 2-3 $\mathrm{mm}$. On the other hand, with neovascularization, some tumor cells can invade into the vascular system, resulting in metastasis. Therefore, attenuating this process can reduce or even prevent tumor growth and metastasis $(25,26)$. Vascular endothelial growth factor (VEGF) family is one of the most important factors in all angiogenic molecules. In general, VEGF refers to VEGFA, which is a highly specific factor that promotes vascular permeability, extracellular matrix degeneration, vascular endothelial cell proliferation, migration and angiogenesis (27). In most RCC patients, there is an excessive expression of VEGFA (28), Therefore, inhibition of VEGF expression is one of the sources that can inhibit RCC angiogenesis. In the present study, miR-218 was found to be overexpressed in RCC cells. This inhibited HUVEC migration, proliferation and tube formation in vitro, and repressed tumor growth and angiogenesis in vivo. Therefore, it was hypothesized that miR-218 may affect the function of HUVECs by regulating the secretion of VEGFA in RCC cells. Indeed, the overexpression of miR-218 in RCC cells reduced the expression and secretion of VEGFA, thereby abrogating the angiogenic ability of the HUVECs. Since the effect of miR-218 on angiogenesis in RCC has not been reported to date, the present study demonstrated for the first time that miR-218 is of great significance in regulating angiogenesis in RCC.

In recent years, it has been found that GAB2 protein is highly expressed in many malignant tumors, and its abnormal expression can mediate the interaction between proteins, affecting various signaling pathways, and regulating tumor cell proliferation, apoptosis and migration (29-32). It has been confirmed that a specific tyrosine residue in the GAB2 protein binds to the P85 subunit in PI3K, activating $\mathrm{PI} 3 \mathrm{~K}$ and producing PIPs that bind to the PH domain in the GAB2 structure. The enhancement of the membrane receptor recruitment of GAB2 results in a positive feedback loop that ultimately leads to GAB2-mediated PI3K/AKT signaling pathway amplification (33). Downstream molecules AKT, mTOR, HIF-1 $\alpha$, and VEGFA are important components of the angiogenic pathway. In the present study, it was demonstrated that miR-218 negatively regulates GAB2 by binding to the specific target sites within the 3'UTR of GAB2, repressing the expression of GAB2 protein. In addition, the phosphorylation status of AKT at Ser473, and the expression of mTOR and HIF-1 $\alpha$ are decreased after overexpression of miR-218. Based on these results, it can be concluded that miR-218 exerts its role in inhibiting RCC angiogenesis by inhibiting the GAB2/PI3K/AKT/mTOR/HIF-1 $\alpha /$ VEGFA pathway (Fig. 7). In addition, research has revealed that the upregulation of HIF-1 $\alpha$ and VEGF mediated by the overexpression of GAB2 in mouse xenograft models promotes tumor angiogenesis (34). This is consistent with the present results, and further proves the validity of the present conclusions. However, this research still has limitations. We did not detect the expression of miR-218 in clinical patient samples. Yet, we will be fully prepared and incorporate clinical patient sample information in future research to further confirm our results.

As endogenous non-coding small RNAs, miRNAs play important roles in a variety of pathophysiological processes. With the advancement of science technology, it is hoped that miRNAs may provide new biomarkers for the diagnosis and prognosis of RCC, and therapeutic targets for the drug development for RCC treatment. Many miRNAs associated with RCC have not been reported to date, and the known miRNAs, such as miR-218, are still not well-researched in regards to RCC development and metastasis. The present study demonstrated that miR-218 significantly repressed RCC tumorigenicity and angiogenesis, providing new insights into the mechanism of RCC carcinogenesis and progression, and suggesting that miRNA-218 may be a target for the treatment of RCC.

\section{Acknowledgements}

Not applicable.

\section{Funding}

This study was supported by grants from the National Natural Science Foundation of China grants (no. 81372736 to YD).

\section{Availability of data and materials}

The datasets used and/or analyzed during the present study are available in the TCGA database.

\section{Authors' contributions}

DW and YD were involved in the design and conceptualization of the study. LM and BG performed the experiments and data generation. JT and XL were involved in the database analysis. QL, MW and WW contributed to the methodology and data curation. LM, BG, JS, XL and YD were involved in the writing, reviewing and editing of the manuscript. JS and XL critically revised the manuscript. YD supervised the study. All authors have read and approved the final manuscript. 


\section{Ethics approval and consent to participate}

The present study was approved by the Ethics Committee of The First Affiliated Hospital of Xi'an Jiaotong University (2013065).

\section{Patient consent for publication}

Not applicable.

\section{Competing interests}

The authors declare that they have no competing interests.

\section{References}

1. Hsieh JJ, Purdue MP, Signoretti S, Swanton C, Albiges L, Schmidinger M, Heng DY, Larkin J and Ficarra V: Renal cell carcinoma. Nat Rev Dis Primers 3: 17009, 2017.

2. Lebacle C, Pooli A, Bessede T, Irani J, Pantuck AJ and Drakaki A: Epidemiology, biology and treatment of sarcomatoid RCC: Current state of the art. World J Urol 37: 115-123, 2019.

3. Siegel R, Ma J, Zou Z and Jemal A: Cancer statistics, 2014. CA Cancer J Clin 64: 9-29, 2014

4. Siegel R, Naishadham D and Jemal A: Cancer statistics, 2012. CA Cancer J Clin 62: 10-29, 2012.

5. Posadas EM and Figlin RA: Systemic therapy in renal cell carcinoma: Advancing paradigms. Oncology (Williston Park) 26: 290-301, 2012.

6. Croce CM: Causes and consequences of microRNA dysregulation in cancer. Nat Rev Genet 10: 704-714, 2009.

7. Gregory RI and Shiekhattar R: MicroRNA biogenesis and cancer. Cancer Res 65: 3509-3512, 2005.

8. Filipowicz W, Bhattacharyya SN and Sonenberg N: Mechanisms of post-transcriptional regulation by microRNAs: Are the answers in sight? Nat Rev Genet 9: 102-114, 2008

9. Bartel DP: MicroRNAs: Target recognition and regulatory functions. Cell 136: 215-233, 2009.

10. Liang H, Ge F, Xu Y, Xiao J, Zhou Z, Liu R and Chen C: miR-153 inhibits the migration and the tube formation of endothelial cells by blocking the paracrine of angiopoietin 1 in breast cancer cells. Angiogenesis 21: 849-860, 2018.

11. Vosgha H, Ariana A, Smith RA and Lam AK: miR-205 targets angiogenesis and EMT concurrently in anaplastic thyroid carcinoma. Endocr Relat Cancer 25: 323-337, 2018.

12. Kinoshita T, Hanazawa T, Nohata N, Kikkawa N, Enokida H, Yoshino H, Yamasaki T, Hidaka H, Nakagawa M, Okamoto Y and Seki N: Tumor suppressive microRNA-218 inhibits cancer cell migration and invasion through targeting laminin-332 in head and neck squamous cell carcinoma. Oncotarget 3: 1386-1400, 2012.

13. Uesugi A, Kozaki K, Tsuruta T, Furuta M, Morita K, Imoto I, Omura $\mathrm{K}$ and Inazawa J: The tumor suppressive microRNA miR-218 targets the mTOR component Rictor and inhibits AKT phosphorylation in oral cancer. Cancer Res 71: 5765-5778, 2011.

14. Tie J, Pan Y, Zhao L, Wu K, Liu J, Sun S, Guo X, Wang B, Gang Y, Zhang Y, et al: MiR-218 inhibits invasion and metastasis of gastric cancer by targeting the Robol receptor. PLoS Genet 6: e1000879, 2010.

15. Small EM and Olson EN: Pervasive roles of microRNAs in cardiovascular biology. Nature 469: 336-342, 2011.

16. Guan B, Wu K, Zeng J, Xu S, Mu L, Gao Y, Wang K, Ma Z, Tian J, Shi Q, et al: Tumor-suppressive microRNA-218 inhibits tumor angiogenesis via targeting the mTOR component RICTOR in prostate cancer. Oncotarget 8: 8162-8172, 2017.
17. Livak KJ and Schmittgen TD: Analysis of relative gene expression data using real-time quantitative PCR and the 2(-Delta Delta C(T)) method. Methods 25: 402-408, 2001.

18. Gao Y, Wu K, Chen Y, Zhou J, Du C, Shi Q, Xu S, Jia J, Tang X, $\mathrm{Li}$, et al: Beyond proliferation: KLF5 promotes angiogenesis of bladder cancer through directly regulating VEGFA transcription. Oncotarget 6: 43791-43805, 2015.

19. Incoronato M, Urso L, Portela A, Laukkanen MO, Soini Y, Quintavalle C, Keller S, Esteller M and Condorelli G: Epigenetic regulation of miR-212 expression in lung cancer. PLoS One 6: e27722, 2011.

20. Leite KR, Tomiyama A, Reis ST, Sousa-Canavez JM, Sañudo A, Camara-Lopes LH and Srougi M: MicroRNA expression profiles in the progression of prostate cancer-from high-grade prostate intraepithelial neoplasia to metastasis. Urol Oncol 31: 796-801, 2013.

21. Grammatikakis I, Gorospe M and Abdelmohsen K: Modulation of cancer traits by tumor suppressor microRNAs. Int J Mol Sci 14: 1822-1842, 2013

22. Venkataraman S, Birks DK, Balakrishnan I, Alimova I, Harris PS, Patel PR, Handler MH, Dubuc A, Taylor MD, Foreman NK and Vibhakar R: MicroRNA 218 acts as a tumor suppressor by targeting multiple cancer phenotype-associated genes in medulloblastoma. J Biol Chem 288: 1918-1928, 2013.

23. Folkman J: What is the evidence that tumors are angiogenesis dependent? J Natl Cancer Inst 82: 4-6, 1990.

24. Karumanchi SA, Merchan J and Sukhatme VP: Renal cancer: Molecular mechanisms and newer therapeutic options. Curr Opin Nephrol Hypertens 11: 37-42, 2002.

25. Folkman J: Angiogenesis in cancer, vascular, rheumatoid and other disease. Nat Med 1: 27-31, 1995.

26. Aishima S, Taguchi K, Sugimachi K, Asayama Y, Nishi H, Shimada M, Sugimachi K and Tsuneyoshi M: The role of thymidine phosphorylase and thrombospondin-1 in angiogenesis and progression of intrahepatic cholangiocarcinoma. Int J Surg Pathol 10: 47-56, 2002.

27. Ferrara N: VEGF and the quest for tumour angiogenesis factors. Nat Rev Cancer 2: 795-803, 2002.

28. Jacobsen J, Grankvist K, Rasmuson T, Bergh A, Landberg G and Ljungberg B: Expression of vascular endothelial growth factor protein in human renal cell carcinoma. BJU Int 93: 297-302, 2004.

29. Wöhrle FU, Daly RJ and Brummer T: Function, regulation and pathological roles of the Gab/DOS docking proteins. Cell Commun Signal 7: 22, 2009.

30. Sattler M, Mohi MG, Pride YB, Quinnan LR, Malouf NA, Podar K, Gesbert F, Iwasaki H, Li S, Van Etten RA, et al: Critical role for Gab2 in transformation by BCR/ABL. Cancer Cell 1: 479-492, 2002.

31. Wang Y, Sheng Q, Spillman MA, Behbakht K and Gu H: Gab2 regulates the migratory behaviors and E-cadherin expression via activation of the PI3K pathway in ovarian cancer cells. Oncogene 31: 2512-2520, 2012.

32. Bentires-Alj M, Gil SG, Chan R, Wang ZC, Wang Y, Imanaka N, Harris LN, Richardson A, Neel BG and Gu H: A role for the scaffolding adapter GAB2 in breast cancer. Nat Med 12: 114-121, 2006.

33. Gu H, Pratt JC, Burakoff SJ and Neel BG: Cloning of p97/Gab2, the major SHP2-binding protein in hematopoietic cells, reveals a novel pathway for cytokine-induced gene activation. Mol Cell 2: 729-740, 1998.

34. Yang Y, Wu J, Demir A, Castillo-Martin M, Melamed RD, Zhang G, Fukunaga-Kanabis M, Perez-Lorenzo R, Zheng B, Silvers DN, et al: GAB2 induces tumor angiogenesis in NRAS-driven melanoma. Oncogene 32: 3627-3637, 2013.

This work is licensed under a Creative Commons Attribution-NonCommercial-NoDerivatives 4.0 International (CC BY-NC-ND 4.0) License. 